\title{
Technikfolgenabschätzung als Schritt zu transformativer Wissenschaft
}

\author{
Technikfolgenabschätzung hat zum Ziel, \\ mögliche Folgen frühzeitig zu erforschen und \\ so Gestaltungsmöglichkeiten aufzuzeigen. \\ Welchen Beitrag kann die Technikfolgenab- \\ schätzung zur Entwicklung einer trans- \\ formativen Wissenschaft leisten? \\ Von Armin Grunwald
}

\begin{abstract}
D e Erfahrung, dass der wissenschaftlich-technische Fortschritt neben seinen unbezweifelbaren Erfolgen auch nicht beabsichtigte und häufig negative Folgen wie z.B. technische Risiken oder Umweltprobleme mit sich bringt, ist eine der Grundmotivationen der Technikfolgenabschätzung (TA).
\end{abstract}

\section{Motivationen der Technikfolgenabschätzung}

In der Ambivalenz des Fortschritts (Grunwald 2010) geht es darum, die möglichen Folgen der Entwicklung und Nutzung von Technik frühzeitig zu erforschen, um gesellschaftliche Gestaltungsoptionen zu eröffnen. Dies gilt in beiden Richtungen, als Frühwarnung vor technikbedingten Gefahren wie auch als Früh erkennung der Chancen, damit Abwägungen von Chancen und Risiken vorgenommen werden können. TA soll die Voraussicht für mögliche Technikfolgen zeitlich wie thematisch systematisch ausweiten, in Abkehr von „wait and see“-Strategien, nach denen erst im Falle des Eintretens unerwarteter negativer Technikfolgen Handlungsbedarf bestehe. Dabei fokussiert TA auf die öffentlich relevante und politische Seite in der Gestaltung des wissenschaftlichen Fortschritts unter Einbeziehung unterschiedlicher Perspektiven, zum Beispiel von Stakeholdern und Betroffenen, und auf alternative Optionen dieser Gestaltung.

Zukunftsentscheidungen sind nach Überzeugung der TA nicht einfach wissenschaftlich entscheidbare oder optimierbare Sachfragen, sondern auch Entscheidungen beispielsweise der Art, in welcher Gesellschaft wir leben wollen, welche Risiken wir einzugehen bereit sind und welche Rolle Technik darin spielen soll. Und diese Entscheidungen sind wert- und positionsabhängig. Dementsprechend will TA ein Denken in transparenten Alternativen befördern, verbunden mit der Erwartung, demokratische Debatte und politische Entscheidungsfindung mit besserem Wissen und transparenter normativer Orientierung versorgen zu können.
Die Anfänge der TA waren von einem Technikdeterminismus geprägt. Danach folgt die wissenschaftlich-technische Entwicklung einer inhärenten Eigendynamik, welche Folgen in Form von Technologien und entsprechenden Produkten und Systemen hervorbringt, an welche die Gesellschaft sich mehr oder weniger anpassen müsse. Ihr bliebe an Handlungsmöglichkeiten nur übrig, darauf zu reagieren und Anpassungsstrategien zu entwickeln. Die Hauptaufgabe der TA in diesem Sinne war prognostisch: Technikfolgen möglichst gut vorherzusehen, damit die Gesellschaft Zeit gewinne, derartige Anpassungs- oder auch Kompensationsstrategien zu entwickeln. Dieser Ansatz wurde aufgegeben, unter anderem wegen der Unsicherheiten des Zukunftswissens und der Erkenntnisse der Technikgeneseforschung, die zu einer Zurückweisung des Determinismus führte.

Dementsprechend trat in der wissenschaftlichen und gesellschaftlichen Diskussion über Technik und Technikfolgen seit den 1990er Jahren der Begriff der Technikgestaltung in den Vordergrund. Insbesondere der niederländische Sozialkonstruktivismus (z.B. Bijker/Law 1994) hat dazu beigetragen, Technik als eine sozial beeinflussbare Größe zu verstehen. TA solle daher die Technikgenese in den Blick nehmen und sich dort aktiv am Prozess der Entstehung und Entwicklung von Technik beteiligen, um bereits an der Wurzel positive Entwicklungen zu verstärken und negative zu verhindern.

\section{Von der Sozialverträglichkeit zur Ko-Evolution}

Bis hierher ist von transformativer Wissenschaft keine Rede gewesen. Im Gegenteil, man kann TA, wie sie bislang beschrieben wurde, auch als Element einer in ihren Grundzügen statischen Gesellschaft verstehen, in denen es nicht um Transformation, sondern um die Einpassung neuer Technik in vorhandene Strukturen, Werte und Wahrnehmungsmuster geht.

Gemeint ist sozialverträgliche Technikgestaltung (Alemann / Schatz 1987), die in einer bestimmten Weise auf das Aufkommen teils gravierender Technikkonflikte reagierte. Die Idee war, bereits in der Technikgestaltung die mutmaßliche spätere Akzeptanz in der Bevölkerung zu berücksichtigen. Technik sollte so ausgelegt werden, dass ihre Verträglichkeit mit den in der Gesellschaft bestehenden Wertstrukturen, zum Beispiel hinsichtlich der Risikobereitschaft, garantiert oder wenigstens wahrscheinlich sei. Dann sollte Technik, so die Erwartung, auf Zustimmung in der Gesellschaft stoßen, weil sie ja zu ihr passen würde. Allerdings, und das ist einer der Gründe für das Scheitern, kann eine Orientierung der Technikentwicklung $\rightarrow$ 
an gegenwärtigen Werten nicht die zukünftige Technikakzeptanz verbürgen, insbesondere bei langen Entwicklungs- und Innovationsprozessen. Werte können sich ändern und die gesellschaftliche Bewertung einer vormals als akzeptabel oder sogar wünschenswert eingestuften Technik kann sich in das Gegenteil verkehren. Noch mehr, Werte, Lebensstile und Akzeptanzmuster können sich gerade auch durch neue Technologien ändern. Gesellschaft ist eben keine statische Größe, in die man neue Technik nur passgenau einfügen muss, sondern verändert sich durch Technik.

Diese Beobachtung hat Anlass gegeben, weder dem früheren Technikdeterminismus noch einer Gestaltungseuphorie nach gesellschaftlichen Werten zu folgen, sondern das Verhältnis von Technik und Gesellschaft als wechselseitige Beeinflussung zu verstehen, als „Ko-Evolution“ (Rip 2007). Freilich, dies hilft nicht gerade bei der Festlegung von Kriterien der Technikbewertung und Technikgestaltung: Einerseits sollen diese Kriterien den Gang der Entwicklung über darauf basierende Bewertungen und Entscheidungen beeinflussen, andererseits können sie selbst von der technischen Entwicklung und von deren gesellschaftlicher Adaptation beeinflusst werden. Hier nun wird klar, dass TA ganz grundsätzlich innerhalb von laufenden Transformationsprozessen arbeiten muss, in denen es keinen archimedischen Punkt gibt.

\section{Strategisches Wissen für Transformation}

Der wissenschaftlich-technische Fortschritt transformiert die Gesellschaft, von der, in der und für die er betrieben wird. Die eingangs genannten Motivationen der TA werden dadurch nicht obsolet, ganz im Gegenteil. Entscheidungen müssen getroffen werden, ob zum Beispiel in Regulierung oder Technikförderung, und sie sollen vorausschauend und reflektiert getroffen werden. Allerdings verschärfen sich die Herausforderungen. TA muss inmitten von laufenden Transformationsprozessen möglichst robustes Wissen für demokratische Debatte und politische Entscheidungsprozesse bereitstellen. Sie hat auf diese Herausforderungen unterschiedlich reagiert. Beispielsweise hat sich der Blick von technischen Artefakten auf sozio-technische Systeme verschoben, vor allem im Infrastrukturbereich. Hier, so etwa bei der Energiewende, ist evident, dass TA Teil eines umfassenden Transformationsprozesses ist und diesen gleichzeitig durch Bereitstellung von Wissen und Orientierung im Prozess mit gestaltet. Allgemeiner ist TA als Nachhaltigkeitsbewertung gar nicht anders als Bestandteil von transformativen Prozessen denkbar, in denen sich erst Schritt für Schritt der Weg in eine nachhaltigere Zukunft erschließt (Grunwald 2007). Strategisches Wissen, bereitgestellt durch TA, ist auf diese Weise Teil eines umfassenderen transformativen Wissens, bestehend aus System-, Zukunfts-, Orientierungs- und Handlungswissen. Die Integration, teils auch bereits die Bereitstellung dieser Wissenselemente bedürfen eines inter- oder transdiziplinären $\mathrm{Zu}$ gangs, um Antworten in den Ambivalenzen des wissenschaftlich-technischen Fortschritts zu entwickeln.
Auf diese Weise kann guten Gewissens die These vertreten werden, dass die Geschichte der TA eine Entwicklung hin zu einem transformativen Selbstverständnis beinhaltet. Sicher ist auch heute nicht jede TA-Studie transformativ und muss es auch nicht sein. Aber das Bewusstsein, Teil eines und gleichzeitig Akteur in einem durch den technischen Fortschritt angetriebenen Transformationsprozess zu sein, ist gewachsen. Die Rezeption von Konzepten wie der „Reflexive Governance“ (Voss et al. 2006) oder Gedanken des „Transition Management“ (Kemp/ Rotmans 2004) belegen dieses.

\section{Technikfolgenabschätzung inmitten der Energiewende}

Technik kann nicht isoliert von ihrem sozialen Kontext betrachtet werden. Das gilt auch und gerade für das Energiesystem. Dieses als reine Technik, als große Maschinerie, anzusehen, die, wenn man mit ihr nicht mehr zufrieden ist, durch eine andere und hoffentlich nachhaltigere Maschinerie ersetzt werden könne, ist zu einfach, ja irreführend.

Bereits das bestehende Energiesystem ist in hohem Maß ein sozio-technisches System (Ropohl 1979), und in einem nach Maßgabe der deutschen Energiewende entstehenden zukünftigen Energiesystem muss das „sozio“ noch stärker betont werden (Grunwald 2012; vgl. das Programm der Helmholtz-Allianz Energy-Trans). Fluktuierende und dezentral organisierte Energieträger müssen integriert werden. Neue Akteure, vor allem viele kleine in das Netz einspeisende Anbieter müssen über Regeln, Verträge, Abmachungen über Rechte und Pflichten, Haftungsfragen etc. sozial und rechtlich eingebunden werden. Verantwortlichkeiten und Zuständigkeiten müssen neu verteilt werden, so übernehmen zum Beispiel Stadtwerke und lokale Initiativen Verantwortung in Bereichen, die bislang eher zentral geregelt waren.

Exzellente und nachhaltige Technik ist somit zwar notwendige, aber nicht hinreichende Bedingung für eine gelingende Energiewende. Es muss eine Neuformierung vieler gesellschaftlicher Handlungsfelder hinzukommen. Und das bedeutet, dass die Energiewende nicht einfach die Substitution alter durch neue Technik, sondern ein Stück weit Selbsttransformation der Gesellschaft ist. Diese Erkenntnis hat weitreichende Folgen, vor allem weil über die Neuformierung gesellschaftlicher Handlungsroutinen ein prognostisches Wissen kaum verfügbar ist. $\mathrm{Ob}$, auf welche Weise und in welchem Umfang ein Demand Side Management zur Systemstabilität praktisch beitragen wird; welche Rolle der zwischenstaatliche europäische Ausgleich spielen wird; wie stark das zukünftige Energiesystem dezentralisiert sein wird und ökonomische Anreizsysteme entwickeln werden - all das ist heute nicht wissbar, sondern muss sich erst herausstellen. Damit kann die Energiewende nicht im Modus klassischer Planung betrieben werden, sondern muss mit großen Unsicherheiten und Offenheiten umgehen. Die beliebte Forderung nach einem Masterplan führt in die Irre. Sie mag psychologisch verständlich sein, ist aber nicht einlösbar. Es sei denn, 
ein solcher Masterplan würde in einem ständigen Lernprozess bestehen, der durch eine grobe Orientierung in Richtung auf nachhaltige Entwicklung orientiert ist, in dem jedoch alles andere im Laufe der Transformation selbst verändert werden kann. Die jeweils nächsten Schritte müssen trotz der Langfristanforderungen der Energiewende inkrementell vorgenommen werden - allerdings nicht in beliebiger, sondern durch die langfristigen Zielvorgaben und das Leitbild der Nachhaltigkeit orientierter Weise. Dabei entstehen neue Konflikte, insbesondere zwischen dem Wunsch nach Flexibilität und Reflexivität einerseits (Voss et al. 2006) und den Anforderungen an Planungssicherheit und Festlegung.

\section{Fazit}

In dieser Konstellation entsteht ein deutlich verändertes Herausforderungsprofil für die TA. Stärker als in anderen Feldern der Ko-Evolution müssen hier zukünftige Technik und Technikfolgen zusammengedacht werden mit zukünftigen gesellschaftlichen Entwicklungen (Grunwald 2012). Beispielsweise lösen sozio-technische Szenarien (vgl. die erwähnte Helmholtz-Allianz), in denen dies explizit zum Programm gemacht wird, frühere Potenzialstudien ab, in denen der erwartete technische Fortschritt in eine mehr oder weniger als statisch vorgestellte Gesellschaft hinein projiziert wurde. Auf diese Weise wird TA selbst zum Medium der Transformation.

\section{Literatur}

Alemann, U. von, Schatz H. (1987): Mensch und Technik. Grundlagen und Perspektiven einer sozialverträglichen Technikgestaltung. Opladen

Bijker, W., Law J. (Hg.) (1994): Shaping Technology/Building Society. Cambridge (Mass.)

Grunwald, A. (2007): Working Towards Sustainable Development in the Face of Uncertainty and Incomplete Knowledge. Journal of Environmental Policy \& Planning Volume 9, Issue 3, S. 245-262.
Grunwald, A. (2010): Technikfolgenabschätzung. Eine Einführung. Berlin, 2. Auflage.

Grunwald, A. (2012): Technikzukünfte in der Energiewende - mehr als Zukünfte der Energietechnik. In: A. Grunwald: Technikzukünfte als Medium gesellschaftlicher Zukunftsdebatten und der Technikgestaltung. Karlsruhe.

Kemp, R., Rotmans, J. (2004): Managing the Transition to Sustainable Mobility. In: B. Elzen, F. Geels and K. Green (Hg., 2004): System Innovation and the Transition to Sustainability: Theory, Evidence and Policy. Cheltenham, UK, S. 137-167.

Rip, A. (2007): Die Verzahnung von technologischen und sozialen Determinismen und die Ambivalenzen von Handlungsträgerschaft im "Constructive Technology Assessment“. In: Dolata, U., Werle, R. (Hg.) (2007): Gesellschaft und die Macht der Technik. Frankfurt/New York, S. 83-106.

Rohracher, H. (2008): Energy systems in transition: contributions from social sciences. Int. J. Environmental Technology and Management, Vol. 9, Nos. 2/3, 2008, 144-161.

Ropohl, G. (1979): Eine Systemtheorie der Technik. Zur Grundlegung der Allgemeinen Technologie. Frankfurt.

Voß, J.-P; Bauknecht, D.; Kemp, R. (Hg, 2006): Reflexive Governance for sustainable development. Cheltenham.

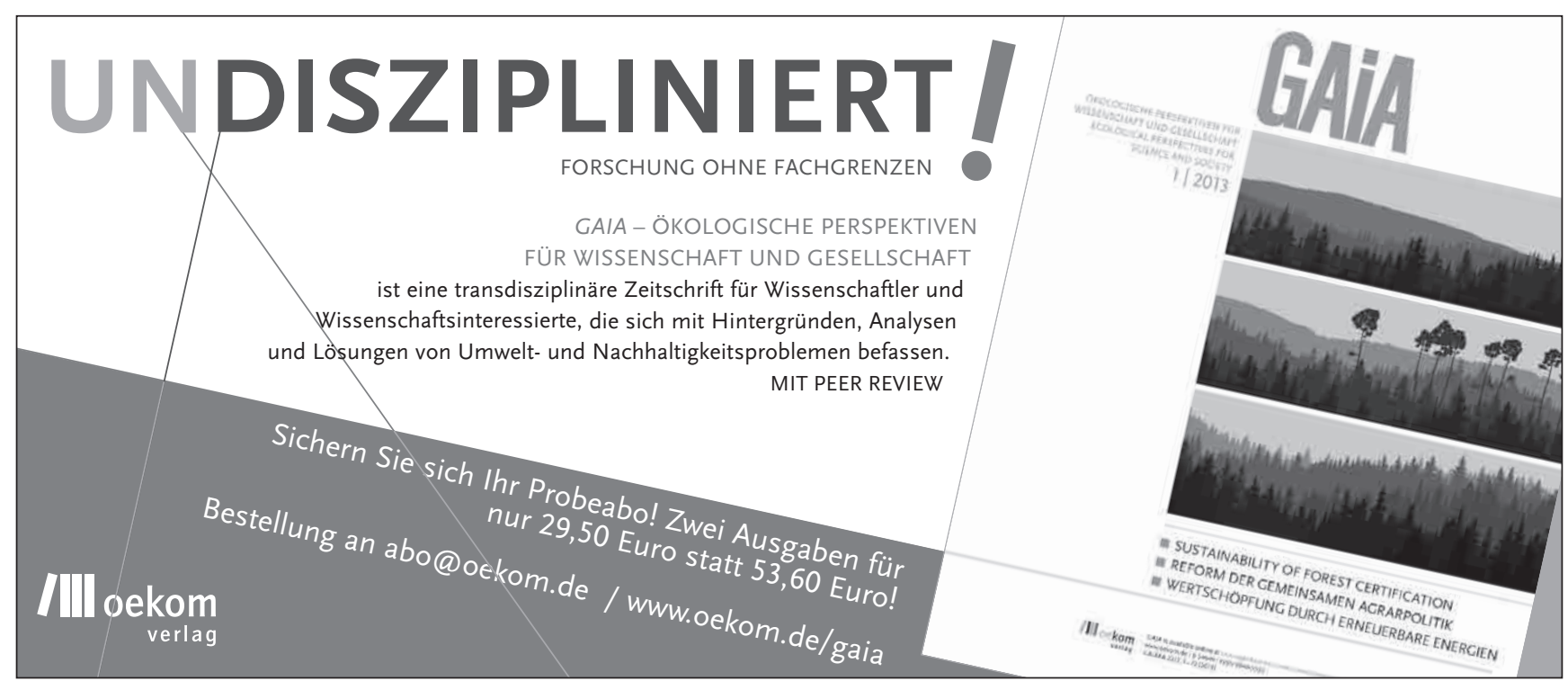


Copyright (C) 2013, IÖW und oekom Verlag. Die Nutzung des Artikels ist Abonnenten von Ökologisches Wirtschaften vorbehalten. Nachdruck und Vervielfältigung des Artikels einschließlich Speicherung und Nutzung auf optischen und elektronischen Datenträgern nur mit Zustimmung der Redaktion von Ökologisches Wirtschaften (http://www.oekologischeswirtschaften.de). 